\title{
Recombinant Hirudin Protects the Vasculature and Myocardium in Atherosclerotic Rats by Inhibiting the p38 MAPK/NF-kB Signaling Pathway
}

\section{Wenping Guo (D 540583116@qq.com )}

Changchun University of Chinese Medicine https://orcid.org/0000-0002-8756-9106

Hongguang Jin

First Affiliated Hospital to Changchun University of Chinese Medicine

\section{Yiqiang Wang}

First Affiliated Hospital to Changchun University of Chinese Medicine

\section{Yongsheng Huang}

First Affiliated Hospital to Changchun University of Chinese Medicine

\section{Xing Zhu}

First Affiliated Hospital to Changchun University of Chinese Medicine

\section{Jiale Wang}

Changchun University of Chinese Medicine

\section{Li Zhang}

Changchun University of Chinese Medicine

\section{Research Article}

Keywords: recombinant hirudin, atherosclerosis, p38 MAPK/NF-KB signaling pathway

Posted Date: September 7th, 2021

DOl: https://doi.org/10.21203/rs.3.rs-866353/v1

License: (c) (i) This work is licensed under a Creative Commons Attribution 4.0 International License. Read Full License 


\section{Abstract}

Recombinant hirudin (r-hirudin) has a good anticoagulant effect and also has a certain inhibitory effect on atherosclerosis (AS), however, its intrinsic mechanism of inhibiting AS is still unclear. In this study, we investigated the mechanism underlying the vascular and myocardial protective effects of r-hirudin in AS rats through animal experiments. A rat AS model was established by high-fat diet feeding combined with common carotid artery balloon injury. The model rats were given low, medium, or high doses of r-hirudin $(0.05,0.1$, or $0.2 \mathrm{mg} / \mathrm{kg} /$ day), simvastatin tablets $(1 \mathrm{mg} / \mathrm{kg} /$ day $)$ and p38 mitogen-activated protein kinase (MAPK) pathway inhibitors (SB203580, $100 \mathrm{mg} / \mathrm{kg} /$ day) by gavage for 8 weeks. The results showed that in AS rats, r-hirudin significantly alleviated pathological changes in the common carotid artery and myocardial tissue; decreased serum total cholesterol (TC), triglyceride (TG) and low-density lipoprotein-cholesterol (LDL-C) levels; increased high-density lipoprotein-cholesterol (HDL-C) levels; decreased serum oxidized low-density lipoprotein (ox-LDL), tumor necrosis factor (TNF)-a, interleukin (IL)-6, IL-1 $\beta$, and endothelin (ET)-1 levels; increased nitric oxide (NO) levels; and decreased p38 MAPK, nuclear factor-kappa B (NF-kB), caspase-9, caspase-3 mRNA and protein expression. This study showed that r-hirudin may protect blood vessels and the myocardium in AS rats by adjusting blood lipid levels and inhibiting the p38 MAPK/NF-KB signaling pathway to exert anti-inflammatory and anti-apoptotic effects and protect the vascular endothelium.

\section{Introduction}

Cardiovascular disease is currently recognized as a major health problem worldwide; its mortality rate remains high, and it seriously endangers the health and reduces the quality of life of patients. Surveys have shown that cardiovascular disease is the leading cause of death and premature death in the Chinese population [1, 2], accounting for $40 \%$ deaths in China. China and India face the greatest challenge related to cardiovascular disease [3]. However, atherosclerosis (AS) is the basis of the pathogenesis of a variety of cardiovascular diseases and is also the underlying cause of cardiovascular accidents [4]. Active intervention in the progression of atherosclerotic lesions and protection of blood vessels and the myocardium from damage induced by AS are the keys for effectively preventing and treating cardiovascular diseases.

The specific mechanism underlying the pathogenesis of AS has not yet been clarified. In recent years, the "endothelial injury response theory", which is supported by most scholars, was proposed; according to this theory, AS is characterized by endothelial dysfunction, the inflammatory response and plaque formation in the arterial vessel wall [5]. Serum endothelin (ET)-1 and nitric oxide (NO) levels are important indicators for evaluating vascular endothelial function, and the ET-1/NO level balance is closely related to the occurrence and development of AS [6]. Furthermore, abnormal lipid metabolism is strongly associated with the occurrence of AS [7, 8]. When blood lipid levels are increased, blood viscosity is increased, and lipids are oxidized to form oxidized low-density lipoprotein (ox-LDL). Ox-LDL strongly stimulates the activation and release of a variety of inflammatory factors, such as tumor necrosis factor (TNF)-a, interleukin (IL)-1 $\beta$, and IL-6, which disrupt the integrity of the vascular intima and stimulate the 
inflammatory immune response in the vascular wall [8]. These inflammatory factors activate the p38 mitogen-activated protein kinase (MAPK) signaling pathway, and p38 MAPK is activated to the phosphorylated form, which promotes the activation and translocation of nuclear transcription factor (NF-kB) to the nucleus; further stimulates the synthesis of a large number of inflammatory factors, such as TNF-a, IL-1 $\beta$, and IL-6; induces a more severe inflammatory response; further damages the vascular intima; and forms a vicious cycle of various processes $[9,10]$. In addition, the massive release of inflammatory factors activates caspase-9, a key promoter of apoptosis; and subsequently activates caspase-3, a downstream apoptotic executioner that promotes the apoptosis of tissue cells and accelerates the progression of atherosclerotic lesions [11, 12].

Statins are currently recognized to treat AS [13]. It has been confirmed that statins can reduce the risk of cardiovascular and cerebrovascular events in individuals of all ages by altering blood lipid levels, but long-term and excessive use of statins leads to various adverse effects, such as rhabdomyolysis, acute renal failure, liver injury and muscle toxicity $[14,15]$. Thus, the clinical use of statins is limited. Recombinant hirudin (r-hirudin) is a genetically engineered product with the same biochemical and pharmacological properties as natural hirudin, which achieves anticoagulant and blood-activating effects mainly by inhibiting thrombin activity [16]. It was confirmed that r-hirudin has a protective effect on blood vessels and the myocardium in patients with AS in the clinic; however, the intrinsic mechanism of this protective effect is still unclear. In this study, we aimed to investigate the possible mechanism by which rhirudin protects blood vessels and the myocardium in AS rats through animal experiments to provide an experimental basis for clinical its application.

\section{Materials And Methods}

\section{Drugs and Reagents}

r-Hirudin was provided by Shanghai Yiyan Biotechnology Co., Ltd., (China, batch number: 8001-27-2). p38 MAPK pathway inhibitor (SB203580) was provided by Shanghai Jizhi Biochemical Technology Co., Ltd., (China, batch number: 124-20-9). Simvastatin tablets were provided by Hangzhou MSD Pharmaceutical Co., Ltd. (China, GYZZ J20130068). Low-molecular-weight heparin sodium (Jipalin) was obtained from Hangzhou Jiuyuan Genetic Engineering Co., Ltd. (China, approval number: GYZZ H19990035). Penicillin sodium was provided by North China Pharmaceutical Co., Ltd., (China, batch number: D0801320).

Total cholesterol (TC) (Nanjing Jiancheng Bioengineering Institute, China, batch number: 20210405), triglyceride (TG) (Nanjing Jiancheng Bioengineering Institute, China, batch number: 20210408), lowdensity lipoprotein-cholesterol (LDL-C) (Nanjing Jiancheng Bioengineering Institute, China, batch number: 20210407) and high-density lipoprotein-cholesterol (HDL-C) kits (Nanjing Jiancheng Bioengineering Institute, China, batch number: 20210407); $\beta$-actin (Proteintech, USA, batch number: 66009-1-Ig), p38 MAPK (Proteintech, USA, batch number: 14064-1-AP), p65NF-KB (Proteintech, USA, batch number: 107451-AP), Caspase-9 (Proteintech, USA, batch number: 10380-1-AP), and Caspase-3 (Proteintech, USA, batch number: 19677-1-AP) antibodies; Ox-LDL, TNF-a, IL-1 $\beta$, IL-6, ET-1 and NO enzyme-linked immunosorbent 
assay (ELISA) kits (Shanghai Optimal Biotechnology Co., Ltd., China, batch number: 202104); a highpurity total RNA rapid extraction kit (Beijing BioTeke Biotechnology Co., Ltd., China, batch number: B027009019); and a BioTeke Super RT kit (Beijing BioTeke Biotechnology Co., Ltd., China, batch number: B020009018) were used. Primers were synthesized by Shanghai Sangon Biological Engineering Co., Ltd.

\section{Animals and Treatments}

Seventy healthy male purebred Sprague-Dawley (SD) rats weighing $280 \sim 300 \mathrm{~g}$ were provided by Liaoning Changsheng Biotechnology Co., Ltd. (SCXK: (Liao) 2015-0001). The experimental rats were housed in the animal room of Jilin University School of Basic Medicine, which was a well-ventilated, quiet room with a controlled temperature of $18 \sim 22^{\circ} \mathrm{C}$ and a controlled humidity of $50 \% \sim 70 \%$. The animals were housed on a $12 \mathrm{~h}$ light/dark cycle and given ad libitum access to water and chow pellets for adaptive feeding for 1 week.

After 1 week of feeding, the rats were randomly divided into seven groups: the control group, AS model group, simvastatin group, low-dose r-hirudin group, medium-dose r-hirudin group, high-dose r-hirudin group, and pathway inhibitor group (10 rats in each group). The common carotid artery was injured with a balloon in all groups except for the control group, in which the external jugular artery was ligated but balloon injury was not induced. After surgery, the animals were given ad libitum access to a normal diet and water for 1 week. Beginning 2 weeks after the operation, the rats in all groups except the sham operation group, which were still given a normal diet, were fed a high-fat diet. At 6 weeks after operation, the low-, medium- and high-dose r-hirudin groups were given the drug at doses of $0.05 \mathrm{mg} / \mathrm{kg} / \mathrm{d}, 0.1$ $\mathrm{mg} / \mathrm{kg} / \mathrm{d}$ and $0.2 \mathrm{mg} / \mathrm{kg} / \mathrm{d}$, respectively; the simvastatin group was given $1 \mathrm{mg} / \mathrm{kg} / \mathrm{d}$ simvastatin tablets; and the pathway inhibitor group was given the p38 MAPK pathway inhibitor SB203580 at a concentration of $100 \mathrm{mg} / \mathrm{kg} / \mathrm{d}$ by gavage. The sham operation group was given an equal volume of normal saline for 8 weeks. At the end of the experiment, the animals were euthanized with carbon dioxide, and samples were taken for appropriate tests.

\section{Histopathological Examination}

Specimens were fixed in $4 \%$ paraformaldehyde, embedded in paraffin, and sectioned with an ultramicrotome at a thickness of $5 \mu \mathrm{m}$. After sectioning, hematoxylin and eosin (HE) and Masson staining were performed, and histomorphological changes were observed under a light microscope.

\section{Determination of Blood Lipid Levels}

Serum samples were placed in a refrigerator at $4^{\circ} \mathrm{C}$ for 30 minutes and then incubated at room temperature for 30 minutes. After the serum was fully thawed, TC, TG, LDL-C and HDL-C levels were determined using kits and a multifunctional microplate reader according to the manufacturer's instructions.

\section{ELISA}


The serum levels of ox-LDL, TNF-a, IL-1 $\beta$, IL-6, ET-1 and NO were measured by ELISA. The ELISA kits were removed from the refrigerator and warmed at room temperature for approximately 30 minutes. The instructions of the kits were followed precisely.

\section{Quantitative Real-Time Polymerase Chain Reaction (qRT-PCR)}

Total RNA was extracted from the rat common carotid artery using a high-purity total RNA rapid extraction kit. Then, total RNA was reverse transcribed into cDNA by qPCR using the BioTeke Super RT Kit. qRT-PCR was performed using Roche Fast Start Universal SYBR Green Master Mix (Rox). The above operations were performed in strict accordance with the manufacturer's instructions. $\beta$-Actin was used as an internal reference gene, and the relative expression of the target gene in each group was measured for comparison. The PCR conditions were as follows: activation at $50^{\circ} \mathrm{C}$ for 2 minutes and $95^{\circ} \mathrm{C}$ for 10 minutes followed by denaturation at $95^{\circ} \mathrm{C}$ for $15 \mathrm{~s}$ and annealing and extension at $60^{\circ} \mathrm{C}$ for 1 minute. $\mathrm{A}$ total of 40 cycles were performed. The specific primer sequences were as follows: p38 MAPK: F, 5'TTGGTCTGTTGGATGTGTTTAC-3', and R, 5'-TGGATTATGTCAGCCGAGTG-3'; NF-kB: F, 5'TTGCTGGTCCCACATAGTTG-3', and R, 5'-ATGTATGTGAAGGCCCATCC-3'; caspase-3: F, 5'GGACTGCGGTATTGAGA-3', and R, 5 '-GGTGCGGTAGAGTAAGC-3'; caspase-9: F, 5'GTGAAGAACGACCTGACT-3', and R, 5'-AGGATGACCACACACACACACCA-3'; $\beta$-actin: F, 5 'CAAGCTTAATCAGG-3', and R, 5 '-ACATGAAGAGAGAGAGAGAGAGGC-3'.

\section{Western Blot Analysis}

The rat common carotid artery was collected, rinsed several times in precooled normal saline, weighed and placed in a tissue homogenizer. Protein was extracted according to the instructions of a tissue cell lysate kit. The protein concentration was determined by the Coomassie brilliant blue method. SDS-PAGE was performed ( $150 \mathrm{~V}, 50 \mathrm{~min})$, and the proteins were transferred to a membrane for $1 \mathrm{~h}$ at $100 \mathrm{~V}$. After transfer, the membrane was washed with TBST 3 times, blocked with skimmed milk powder for $2 \mathrm{~h}$, washed with TBST, incubated with primary antibody overnight at $4^{\circ} \mathrm{C}$, washed with TBST 3 times, incubated with secondary antibody at room temperature for $1 \mathrm{~h}$, and washed with TBST 3 times. Color development was performed with a high-sensitivity ECL chromogenic luminescence kit according to the manufacturer's instructions. $\beta$-Actin was used as an internal reference protein, gray values were analyzed and determined using a gel imaging system, and the relative expression of each protein was calculated as the gray value of the sample band/the gray value of the $\beta$-actin band for quantitative analysis. Each experiment was repeated three times.

\section{Statistical Analysis}

Statistical analysis was performed with SPSS 21.0 and GraphPad Prism 6.0 software. All data are presented as the mean \pm standard deviation. One-way analysis of variance and the least significant post hoc difference test were used to evaluate the significance of differences. $\mathrm{P}<0.05$ was considered statistically significant. 


\section{Results}

\section{r-Hirudin Can Inhibit Damage to the Common Carotid Artery and Myocardial Tissue in AS Rats}

The results of HE staining showed that the structure of common carotid artery was normal, the intima was continuous, and smooth muscle cells and elastic fibers were neatly arranged in the control group; in contrast, in the model group, intimal thickening was observed in common carotid artery lesions, the structure was significantly disrupted, cholesterol deposition was observed, a large number of foam cells and a small number of infiltrating lymphocytes were present, smooth muscle cells were significantly disorganized, elastic fibers were arranged in a wavy pattern, and some elastic fibers were broken. The pathological changes in the common carotid artery were significantly alleviated in the simvastatin group compared with the model group and in the p38 MAPK pathway inhibitor group compared with the model group. The intima of the common carotid artery was basically smooth, the space between elastic fiber lamellae was nonsignificantly increased, smooth muscle cells were arranged neatly, and there were no obvious foam cells or lymphocytes. The pathological changes in the common carotid artery were inhibited to a certain extent in the r-hirudin groups compared with the model group, and this inhibitory effect was dose-dependent, with the high-dose r-hirudin having the most obvious effect (Fig. 1). HE staining of myocardial tissue revealed neatly arranged myocardial cells, clear transverse striations of basically consistent size and shape; clear and discernible nuclei and cytoplasm and there were no obvious inflammatory infiltration or necrotic lesions in the control group. In contrast, in the model group, myocardial cells were irregularly arranged, and the cell structure was destroyed, as indicated mainly by myocardial cell acidophilic degeneration and vacuolar degeneration. The nuclear volume was decreased; the nuclei were pyknotic; the cytoplasm volume was reduced or the cytoplasm was absent; some myofilaments were broken and dissolved; there were infiltrating inflammatory cells; and there were stripped, reticular and irregular collagen fibers between the myocardium. The pathological changes in myocardial tissue were significantly alleviated in the simvastatin group and p38 MAPK pathway inhibitor group compared with the model group; the arrangement of myocardial cells was relatively regular, and only some cell structures were destroyed; some myofilaments showed breakage and dissolution, and there was a small amount of inflammatory cell infiltration. The pathological changes in myocardial tissue were inhibited to a certain extent in the r-hirudin groups compared with the model group, and the effects were dose-dependent, with the high dose of r-hirudin showing the most obvious effect, followed by the medium and low doses. The above results showed that $r$-hirudin inhibited damage to the common carotid artery and myocardium in AS rats (Fig. 2).

\section{r-Hirudin Can Inhibit Fibrosis of the Common Carotid Artery and Myocardium in AS Rats}

The results of Masson staining showed that common carotid arteries from the control group showed red muscle fibers but no obvious collagen fibers. In contrast, there was significant intimal hyperplasia in common carotid arteries from the model group, as blue collagen fibers were mainly observed. Compared 
with the control group, the simvastatin, the low-dose r-hirudin, medium-dose r-hirudin, high-dose r-hirudin, and p38 MAPK pathway inhibitor groups showed partial hyperplasia of the intima and collagen fibers in some areas, the number of collagen fibers was significantly reduced and intimal hyperplasia was alleviated in these groups compared with the model group. Among these groups, the simvastatin group, the high-dose r-hirudin group and the p38 MAPK pathway inhibitor group showed significant improvement, the middle-dose r-hirudin group showed moderate improvement, and the low-dose r-hirudin group showed only partial improvement (Fig. 3). Masson staining of myocardial tissue showed that there was very little collagen deposition in the control group and that there was almost no collagen deposited between myocardial cells and around blood vessels. In the model group, there was obvious collagen fiber deposition in the myocardial interstitium, and the collagen fibers were striped or mesh-like and disorderly arranged. Collagen deposition between myocardial cells was alleviated to varying degrees in the simvastatin group, the low-dose r-hirudin group, the medium-dose r-hirudin group the high-dose r-hirudin group, and the p38 MAPK pathway inhibitor group compared with the model group. Among these groups, the simvastatin group, the high-dose r-hirudin group and the p38 MAPK pathway inhibitor group showed significant improvement, the middle-dose r-hirudin group showed moderate improvement, and the lowdose r-hirudin group showed only partially improvement. The above results showed that r-hirudin can dose-dependently inhibit fibrosis in the common carotid artery and myocardium in AS rats (Fig. 4).

\section{r-Hirudin Can Alter Blood Lipid Level in AS Rats}

Compared with those in the control group, the serum TC, TG, and LDL-C levels in the model group were significantly increased $(P<0.001)$, and the level of HDL-C was significantly decreased $(P<0.001)$. Compared with those in the model group, the serum TC, TG and LDL-C levels in the simvastatin group and the pathway inhibitor group were extremely significantly reduced $(P<0.001)$, and the HDL-C level was significantly increased $(P<0.05)$. Compared with those in the model group, the serum $T C, T G, L D L-C$ levels in rats in the low-, medium-, and high-dose r-hirudin groups were decreased, with the decrease being the most significant in the high-dose r-hirudin group $(P<0.001)$. The level of HDL-C in the low-, mediumand high-dose r-hirudin groups increased, but the increase in the low-dose r-hirudin group was not statistically significant $(P>0.05)$. The HDL-C level in the middle-dose r-hirudin group increased significantly $(P<0.05)$. The level of HDL-C in the high-dose r-hirudin group increased significantly $(P<$ 0.001), and r-hirudin had a dose-dependent effect (Table 1). Compared with that in the control group, the serum ox-LDL level in the model group was significantly increased $(P<0.05)$. Compared with that in the model group, the serum ox-LDL level in the simvastatin group and the pathway inhibitor group was significantly reduced $(P<0.05)$, and the serum ox-LDL level in the low- and medium-dose r-hirudin groups was significantly reduced $(P<0.05)$. Serum ox-LDL levels in the high-dose $r$-hirudin group were extremely significantly reduced $(P<0.001)$, and the drug had a dose-dependent effect (Fig. 5). 
Table 1

Lipid levels in rats from the different groups

\begin{tabular}{|c|c|c|c|c|}
\hline Study group & TC & TG & LDL-C & HDL-C \\
\hline Control & $1.20 \pm 0.29$ & $0.80 \pm 0.13$ & $0.34 \pm 0.05$ & $0.90 \pm 0.12$ \\
\hline Model & $3.14 \pm 0.42 *$ & $3.06 \pm 0.32 *$ & $1.44 \pm 0.19 *$ & $0.66 \pm 0.10 *$ \\
\hline Simvastatin & $1.71 \pm 0.41^{\text {枢 }}$ & $1.81 \pm 0.43^{\# \rrbracket}$ & $0.89 \pm 0.38^{\text {\#囚 }}$ & $0.89 \pm 0.22^{\#}$ \\
\hline Low-dose $\mathrm{r}-\mathrm{H}$ & $2.58 \pm 0.46^{\#}$ & $2.57 \pm 0.60^{\#}$ & $0.84 \pm 0.28^{\# \rrbracket}$ & $0.72 \pm 0.11$ \\
\hline Middle-dose r-H & $2.02 \pm 0.32^{\# \boxplus ~}$ & $2.50 \pm 0.54^{\#}$ & 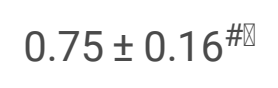 & $0.80 \pm 0.10^{\#}$ \\
\hline High-dose r-H & $1.72 \pm 0.10^{\text {\# }}$ & $1.65 \pm 0.39$ \#\# & $0.56 \pm 0.12^{\text {\#囚 }}$ & $0.90 \pm 0.13^{\text {\#囚 }}$ \\
\hline Inhibitor & $2.83 \pm 0.39$ & $2.79 \pm 0.28$ & $1.35 \pm 0.29$ & $0.71 \pm 0.12$ \\
\hline \multicolumn{5}{|c|}{ Values are mean \pm standard deviation; } \\
\hline \multicolumn{5}{|c|}{$\begin{array}{l}T C \text {, total cholesterol; } T G \text {, triglyceride; } L D L-C \text {, low-density lipoprotein-cholesterol; } H D L-C \text {, high-density } \\
\text { lipoprotein-cholesterol. Compared with the control group, }{ }^{\star} P<0.001 \text {; compared with the model group, } \\
P<0.05,{ }^{\square} \mathrm{P}<0.001 \text {. }\end{array}$} \\
\hline
\end{tabular}

\section{r-Hirudin Can Reduce Serum Inflammatory Factor Levels in AS Rats}

Compared with those in the control group, the levels of serum TNF-a, IL-1 $\beta$, and IL- 6 in the model group were significantly increased $(P<0.01)$. Compared with those in the model group, the serum TNF- $a$ and IL$1 \beta$ levels in the simvastatin group and the pathway inhibitor group were extremely significantly reduced $(P<0.01)$, and the IL-6 level was significantly reduced $(P<0.05)$. Compared with those in the model group, the levels of serum TNF-a, IL-1 $\beta$ and IL- 6 in the low-dose r-hirudin group were not significantly different (P $>0.05$ ). The serum levels of TNF- $\alpha$, IL-1 $\beta$, and IL- 6 in the middle-dose r-hirudin group decreased. The serum levels of TNF- $a$, IL- $1 \beta$ and IL- 6 in the high-dose r-hirudin group decreased significantly, but the level of TNF-a decreased more significantly $(P<0.01)$ (Fig. 6).

\section{r-Hirudin Can Regulate the Balance of Serum ET-1/NO Levels in AS Rats}

Compared with that in the control group, the serum ET-1 level in the model group was significantly increased $(P<0.01)$, and the NO level was significantly decreased $(P<0.01)$. Compared with those in the model group, serum ET-1 levels in the simvastatin, low-dose r-hirudin, medium-dose r-hirudin and highdose r-hirudin, and pathway inhibitor groups were extremely significantly reduced $(P<0.01)$. Serum NO levels in the simvastatin group, high-dose r-hirudin group and pathway inhibitor group were significantly 
increased $(P<0.05)$; however, serum NO levels in the low- and medium-dose r-hirudin groups were not significantly changed $(P>0.05)$ (Fig. 7).

\section{r-Hirudin Inhibits the Inflammatory Response in the Common Carotid Artery in AS Rats by Decreasing the mRNA and Protein Expression Levels of p38 MAPK/NF-KB}

Compared with those in the control group, the mRNA and protein expression levels of p38 MAPK/NF-KB in the common carotid artery in the model group were extremely significantly increased $(P<0.01)$.

Compared with that in the model group, the mRNA expression of p38 MAPK/NF-kB in the common carotid artery in the simvastatin group and the pathway inhibitor group was extremely significantly reduced $(P<0.01)$. Compared with those in the model group, the mRNA expression levels of $p 38$ MAPK/NF-KB in the low-, medium- and high-dose r-hirudin groups were decreased, with the decrease being the most significant in the high-dose r-hirudin group $(P<0.01)$.

Compared with that in the model group, the protein expression of p38 MAPK/NF-KB in the common carotid artery in the simvastatin group and the pathway inhibitor group was extremely significantly reduced $(P<0.01)$. Compared with that in the model group, the protein expression level of p38 MAPK in the low- and medium-dose r-hirudin groups was decreased, but the difference was not statistically significant $(P>0.05)$; furthermore the protein expression level of p38 MAPK in the high-dose r-hirudin group was extremely significantly reduced $(P<0.01)$. The protein expression level of NF-KB in the lowand medium-dose r-hirudin groups was significantly decreased $(P<0.05)$, and the protein expression level of NF-KB in the high-dose r-hirudin group was extremely significantly decreased $(P<0.01)$ (Fig. 8).

\section{r-Hirudin Inhibits Apoptosis of Common Carotid Artery Cells in AS Rats by Decreasing the mRNA and Protein Expression Levels of caspase-9/caspase-3}

Compared with those in the control group, the mRNA and protein expression levels of caspase-9/caspase3 in the common carotid artery tissue in the model group were increased, and the difference was significant $(P<0.05$ or $P<0.01)$.

Compared with that in the model group, the mRNA expression of Caspase-9/Caspase 3 in the common carotid artery in the simvastatin group and the pathway inhibitor group was significantly reduced $(\mathrm{P}<$ 0.05). Compared with those in the model group, the mRNA expression levels of caspase-9/caspase- 3 in the common carotid artery in the low-, medium-, and high-dose r-hirudin groups were decreased. However, compared with those in the model group, caspase- 9 mRNA expression levels were not statistically 
different in the low- and medium-dose r-hirudin groups $(P>0.05)$, while caspase-9/caspase-3 mRNA expression levels were significantly reduced in the high-dose r-hirudin group $(P<0.05)$.

Compared with that in the model group, the protein expression level of caspase- $9 /$ caspase- 3 in the common carotid artery in the simvastatin group and the pathway inhibitor group was decreased, and the difference was significant $(P<0.05$ or $P<0.01)$. Compared with that in the model group, the protein expression level of caspase- 9 /caspase-3 in the low-, medium-, and high-dose $r$-hirudin groups was decreased, and the effect was dose-dependent, with the decrease being the most significant in the highdose group (Fig. 9).

\section{Discussion}

AS is characterized by intimal atheromatous lesions in large and medium arteries, and its specific pathogenesis is still unclear and cannot be fully explained by a single theory. However, there is evidence that inflammation-mediated endothelial injury is closely related to AS [17]. The MAPK signaling pathway is an important pathway that mediates the transmission of information through extracellular signals, intracellular signals and nuclear signals, and the MAPK family consists of p38 MAPK, extracellular signalregulated kinase (ERK1/2) and stress-activated protein kinase/c-Jun N-terminal kinase (JNK). Each MAPK is activated by a specific MAPK kinase (MAPKK), which in turn is activated by MAPKK kinase. In the absence of stimulation, the MAPK signaling pathway is quiescent, and in response to stimuli such as inflammatory factors, proteins in this pathway undergo cascade phosphorylation and are activated [18]. As a member of the MAPK family, p38 MAPK is an important signaling molecule involved in inflammatory responses and apoptosis, among other processes $[19,20]$. When the blood lipid concentration increases, blood viscosity increases, and lipids are easily oxidized to ox-LDL. The formation of a massive amount of ox-LDL causes direct damage to vascular endothelial cells and endothelial dysfunction and makes it easier for lipids and monocytes to be deposited in the blood in the subendothelial space, further resulting in the formation of foam cells and thereby initiating AS pathophysiology [21]. NO is a powerful vasodilator, whereas ET-1 is considered an endogenous vasoconstrictor. NO and ET-1 serve as important indicators of endothelial function; when the balance between the levels of the two molecules is disrupted, the secretion of NO is inhibited, while the secretion of ET-1 is increased $[6,22]$. On the other hand, inflammatory factors are robustly activated and released in response to ox-LDL, as demonstrated in numerous in vivo and in vitro experiments [23,24]. In response to inflammatory factors, p38 MAPK can further activate its downstream factor NF-kB by phosphorylating it, and administration of p38 MAPK pathway inhibitors to experimental animals significantly inhibits the expression of p-p38 MAPK and NF-KB [25]. It has been confirmed [26] that inflammatory factors such as TNF- $\alpha$ can activate the $\mathrm{p} 38$ MAPK/NF-KB signaling pathway. When NF-KB is activated, it may in turn further stimulate the p38 MAPK signaling pathway through activated molecules, including TNF-a, IL-1 $\beta$ and IL-6 [27]. This creates a vicious cycle through which the inflammatory response is rapidly amplified. Thus, there is a complex network of mutual activation between p38 MAPK and NF-KB, which ultimately leads to the mutual activation of the two molecules, which mediates the inflammatory response and may be involved in the development of AS. Moreover, upon stimulation by numerous inflammatory factors, the 
activated p38 MAPK/NF-KB signaling pathway can, through another pathway, activate its downstream factor caspase-9 [28]. Caspase-9 is a key promoter of apoptosis, and when Caspase-9 is activated, caspase-3, a downstream key executor of apoptosis, is also activated, accelerating apoptosis and further accelerating the pathological process of AS [28-30]. Nie Nana et al confirmed through in vitro experiments that the addition of p38 MAPK pathway inhibitors to TNF-a-treated cells can effectively improve cell survival and reduce apoptosis [26].

Recombinant hirudin, a novel drug obtained by genetic engineering, has a similar function to hirudin, but it causes less adverse effects. Some studies have confirmed [16,31], and r-hirudin has good anticoagulant and blood-activating effects. It is often used in clinical practice for the treatment of a variety of diseases including cardiovascular diseases $[32,33]$ and has achieved good results.

In our study, a rat model of AS was established by high-fat diet feeding combined with common carotid artery balloon injury. Following the successful establishment of the rat AS model, the rats were randomly divided into groups, and each group of rats received a different intervention. The experimental results showed that there were significant pathological changes in the common carotid artery and myocardial tissue in the model group compared with the control group. The results showed that a high-fat diet combined with balloon injury indeed led to AS, which in turn damaged the common carotid artery and myocardial tissue. At present, high-fat diet feeding combined with balloon injury is a commonly used method to establish AS animal models [34], and our model was successfully established. Compared with those in the model group, the histopathological changes in the common carotid artery and myocardium in the simvastatin group and pathway inhibitor group were significantly improved. Statins have protective effects on blood vessels and the myocardium in many ways, including by exerting anti-inflammatory effects, protecting endothelial function, and altering blood lipid levels [35]. Some studies have also confirmed that p38 MAPK pathway inhibitors have some protective effects on blood vessels and the myocardium in AS rats [36], which may be related to inhibition of various subsequent reactions mediated by this pathway. This is consistent with our experimental results. In rats, the alleviating effect of r-hirudin on the pathological changes in the common carotid artery and myocardium gradually improved with increasing concentration, which indicated that r-hirudin can protect the common carotid artery and myocardium in AS rats in a dose-dependent manner. The results of blood lipid and serum ox-LDL level determination showed that the serum TC, TG, LDL-C and ox-LDL levels were significantly increased, while the HDL-C level was significantly decreased in the model group compared with the control group. This shows that our high-fat diet feeding protocol had a robust effect and that the model animals met the blood lipid level requirements for AS. Compared with those in the model group, serum TC, TG, LDL-C and ox-LDL levels in the simvastatin group were significantly decreased, while the HDL-C level was significantly increased. The above experimental results are in accordance with the ability of statins to alter lipid levels [37]. Compared with those in the model group, the serum TC, TG, LDL-C, HDL-C and oxLDL levels in the pathway inhibitor group showed no significant changes, as the differences were not statistically significant $(P>0.05)$. Thus, pathway inhibitors are not associated with changes in lipid levels. The p38 MAPK pathway inhibitor we employed was SB203580, which inhibits the activation and phosphorylation of p38 MAPK and blocks the subsequent reaction [38,39] but does not alter lipid levels. 
After administration of different concentrations of r-hirudin to AS rats, serum TC, TG, LDL-C and ox-LDL levels were significantly decreased. From our experimental results, it can be seen that the degree of reduction in the levels of the above indicators was related to the concentration of $r$-hirudin. The higher the concentration was, the greater the degree of reduction. However, there was no significant difference in HDL-C level between the low-dose r-hirudin group and the model group, and the effect of high-dose rhirudin was inferior to that of simvastatin. This shows that $r$-hirudin has advantages in reducing serum TC, TG, LDL-C and ox-LDL levels. The ELISA results showed that the levels of serum TNF-a, IL-1 $\beta$, IL-6, ET1 , and NO were significantly changed in the model group compared with the control group. TNF- $a, \mathrm{IL}-1 \beta$, and IL- 6 levels were significantly increased. TNF- $a$, IL- $1 \beta$, and IL- 6 are all recognized inflammatory factors [40-42] thus, the results indicate that a severe inflammatory response occurred in the vascular intima of rats in the model group and that AS is closely related to inflammation. Serum ET-1 levels were similarly increased, while NO levels were decreased in model rats. Some studies have confirmed that ET-1 and NO are important indicators for evaluating vascular endothelial function and that when the vascular endothelium is injured, ET-1 content increases and NO content decreases [43]. Our experimental results are consistent with those of previous studies and indicated that vascular endothelial function was severely impaired in rats in the model group and that the inflammatory response in the vascular intima may have mediated endothelial injury. Compared with those in the model group, the serum levels of TNFa, IL-1 $\beta, I L-6$ and ET-1 in the simvastatin group and pathway inhibitor group were significantly decreased, while the NO level was significantly increased. This result suggests that simvastatin and pathway inhibitors may have an inhibitory effect on the inflammatory process that mediates the development of AS and that vascular endothelial function is protected in AS rats. After administration of different concentrations of r-hirudin to AS rats, the levels of the above indicators were changed to different extents, and the change trend was basically the same as that of simvastatin group and pathway inhibitor group; however, the high concentration of r-hirudin had more robust effects. This shows that r-hirudin can inhibit the inflammatory reaction in the vascular intima and protect endothelial function in AS rats and that the effect of a high dose is more obvious. The qRT-PCR and Western blot results showed that the mRNA and protein expression levels of p38 MAPK/NF-KB in the common carotid arteries of rats were significantly increased in the model group compared with the control group, which indicated that the p38 MAPK/NF$\mathrm{KB}$-mediated inflammatory response may be involved in the development of AS and that these processes are closely related to each other. Similarly, both the mRNA and protein expression levels of caspase9/caspase-3 were significantly increased. This result suggests that p38 MAPK/NF-KB-mediated downstream apoptotic mechanisms may be activated in response to numerous inflammatory factors [44, 45] and that vascular intimal cells undergo apoptosis; thus, this process is also involved in the development of AS. Compared with those in the model group, the mRNA and protein expression levels of p38 MAPK/NF-KB in the common carotid artery in the simvastatin group and the pathway inhibitor group were significantly reduced, which indicated that both simvastatin and pathway inhibitors can effectively inhibit the mRNA and protein expression of p38 MAPK/NF-KB and further inhibit the inflammatory response mediated by this pathway. We also showed that the mRNA and protein expression levels of p38 MAPK were more significantly decreased in the pathway inhibitor group than in the other groups and that the levels in the pathway inhibitor group were similar to those in the control group. This result indicated

Page $12 / 23$ 
that the p38 MAPK pathway inhibitor SB203580 more specifically inhibited p38 MAPK expression. Compared with those in the model group, the mRNA and protein expression levels of caspase-9/caspase3 in the common carotid artery in the simvastatin group and the pathway inhibitor group were also significantly reduced, which indicated that the subsequent apoptotic process mediated by p38 MAPK/NFKB was also significantly inhibited and that simvastatin and pathway inhibitors had certain anti-apoptotic effects. Studies have confirmed the anti-inflammatory and anti-apoptotic effects of simvastatin [46, 47], which is consistent with the experimental results we obtained. Our experimental results showed that caspase-9/caspase-3 mRNA and protein expression levels were decreased in the pathway inhibitor group, which may have been due to the inhibition of p38 MAPK phosphorylation by pathway inhibitors, which in turn inhibited the downstream apoptotic process. r-Hirudin was administered to AS rats at different concentrations, and our experimental results showed that low-dose r-hirudin did not have a significant effect on the mRNA and protein expression of p38 MAPK/NF-KB and caspase-9/caspase-3, medium-dose r-hirudin had a moderate inhibitory effect on the expression of these mRNAs and proteins, while highdose r-hirudin had the most significant inhibitory effect on the expression of these mRNAs and proteins, which was basically the same as that of simvastatin and the pathway inhibitor. This indicated that $r-$ hirudin inhibits the inflammatory reaction process mediated by p38 MAPK/NF-KB and the apoptosis process mediated by Caspase- $9 /$ Caspase- 3 in a dose-dependent manner; the higher the concentration is, the stronger the inhibitory effect.

In conclusion, r-hirudin may protect blood vessels and the myocardium in AS rats through the regulation of blood lipid levels, inhibition of p38 MAPK/NF-KB signaling pathway-mediated inflammation and apoptosis, and protection of vascular endothelial function to prevent and treat AS.

\section{Declarations}

\section{Ethical Approval}

All animal experiments were approved by the Animal Ethics Committee of Changchun University of Chinese Medicine (Changchun, China) (approval no. 2021091) and were performed according to the Standard Operating Procedures for Laboratory Animal Center of Changchun University of Chinese Medicine.

\section{Consent for Publication}

All contributing authors are aware of and agree to the submission of this manuscript.

\section{Availability of Data and Materials}

All data and materials are fully available without restriction.

\section{Conflict of Interest}

The authors declare no conflict of interest. 


\section{Funding}

The study was supported by the Education Department of Jilin Province (No. JJKH20200884KJ).

\section{Authors' Contributions}

Conceptualization: Wenping Guo, Yongsheng Huang.

Data curation: Jiale Wang, Li Zhang.

Formal analysis: Wenping Guo.

Investigation: Yiqiang Wang, Xing Zhu.

Methodology: Hongguang Jin, Yiqiang Wang, Xing Zhu.

Project administration: Hongguang Jin.

Resources: Wenping Guo, Hongguang Jin.

Supervision: Yongsheng Huang.

Writing - original draft: Wenping Guo, Hongguang Jin.

Writing - review \& editing: Wenping Guo, Hongguang Jin.

\section{Acknowledgements}

We acknowledge the financial support of the Education Department of Jilin Province (No. JJKH20200884KJ).

\section{References}

1. Zhou, M. et al. 2016. Cause-specific mortality for 240 causes in China during 1990-2013: a systematic subnational analysis for the Global Burden of Disease Study 2013. Lancet (London, England) 387(10015), 251-272.

2. Yang, G. et al. 2013. Rapid health transition in China, 1990-2010: findings from the Global Burden of Disease Study 2010. Lancet (London, England) 381(9882), 1987-2015.

3. Zhao D, Liu J, Wang M, Zhang X, Zhou M. 2019. Epidemiology of cardiovascular disease in China: current features and implications. Nat Rev Cardiol. 16(4), 203-212.

4. Sun LF, An DQ, Niyazi GL, Ma WH, Xu ZW, Xie Y. 2018. Effects of Tianxiangdan Granule treatment on atherosclerosis via NF-KB and p38 MAPK signaling pathways. Mol Med Rep. 17(1), 1642-1650.

5. Wu Y, Wang F, Fan L, Zhang W, Wang T, Du Y, Bai X. 2018. Baicalin alleviates atherosclerosis by relieving oxidative stress and inflammatory responses via inactivating the NF-KB and p38 MAPK 
signaling pathways. Biomed Pharmacother. 97, 1673-1679.

6. Tao L, Nie Y, Wang G, Ding Y, Ding J, Xiong F, Tang S, Wang Y, Zhou B, Zhu H. 2018. All-trans retinoic acid reduces endothelin-1 expression and increases endothelial nitric oxide synthase phosphorylation in rabbits with atherosclerosis. Mol Med Rep. 17(2), 2619-2625.

7. Hu Y, Sun B, Liu K, Yan M, Zhang Y, Miao C, Ren L. 2016. Icariin Attenuates High-cholesterol Diet Induced Atherosclerosis in Rats by Inhibition of Inflammatory Response and p38 MAPK Signaling Pathway. Inflammation 39(1), 228-236.

8. Wolf D, Ley K. 2019. Immunity and Inflammation in Atherosclerosis. Circ Res. 124(2), 315-327.

9. Raggi P, Genest J, Giles JT, Rayner KJ, Dwivedi G, Beanlands RS, Gupta M. 2018. Role of inflammation in the pathogenesis of atherosclerosis and therapeutic interventions. Atherosclerosis 276, 98-108.

10. Wei Q, Tu Y, Zuo L, Zhao J, Chang Z, Zou Y, Qiu J. 2020. MiR-345-3p attenuates apoptosis and inflammation caused by oxidized low-density lipoprotein by targeting TRAF6 via TAK1/p38/NF-kB signaling in endothelial cells. Life Sci. 241, 117142.

11. Yang Q, Wang C, Jin Y, Ma X, Xie T, Wang J, Liu K, Sun H. 2019. Disocin prevents postmenopausal atherosclerosis in ovariectomized LDLR-/- mice through a PGC-1a/ERa pathway leading to promotion of autophagy and inhibition of oxidative stress, inflammation and apoptosis. Pharmacol Res. 148, 104414.

12. Brentnall M, Rodriguez-Menocal L, De Guevara RL, Cepero E, Boise LH. 2013. Caspase-9, caspase-3 and caspase-7 have distinct roles during intrinsic apoptosis. BMC Cell Biol. 14, 32.

13. Kazi D. S., Penko J. M., Bibbins-Domingo K. 2017. Statins for Primary Prevention of Cardiovascular Disease: Review of Evidence and Recommendations for Clinical Practice. Med Clin North Am. 101(4), 689-699.

14. Horodinschi R. N., Stanescu A. M. A., Bratu O. G., Pantea Stoian A, Radavoi D. G., Diaconu C. C. 2019. Treatment with Statins in Elderly Patients. Medicina (Kaunas) 55(11), 721.

15. Liu A, Wu Q, Guo J, Ares I, Rodríguez JL, Martínez-Larrañaga MR, Yuan Z, Anadón A, Wang X, Martínez MA. 2019. Statins: Adverse reactions, oxidative stress and metabolic interactions. Pharmacol Ther. 195, 54-84.

16. Nowak G. 2002. Pharmacology of recombinant hirudin. Semin Thromb Hemost. 28(5), 415-424.

17. Libby P. 2012. Inflammation in atherosclerosis. Arterioscler Thromb Vasc Biol. 32(9), 2045-51.

18. Reustle A, Torzewski M. 2018. Role of p38 MAPK in Atherosclerosis and Aortic Valve Sclerosis. Int J Mol Sci. 19(12), 3761.

19. Bonney EA. 2017. Mapping out p38MAPK. Am J Reprod Immunol. 77(5), 10.1111/aji.12652.

20. Chang SY, Sun RQ, Feng M, Li YX, Wang HL, Xu YM. 2018. BML-111 inhibits the inflammatory response and apoptosis of renal tissue in rats with hemorrhagic shock by inhibiting the MAPK pathway. Eur Rev Med Pharmacol Sci. 22(11), 3439-3447. 
21. Pirillo A, Norata GD, Catapano AL. 2013. LOX-1, OxLDL, and atherosclerosis. Mediators Inflamm. 2013, 152786.

22. Nishiyama SK, Zhao J, Wray DW, Richardson RS. 2017. Vascular function and endothelin-1: tipping the balance between vasodilation and vasoconstriction. J Appl Physiol (1985). 122(2), 354-360.

23. Pang JL, Wang JW, Hu PY, Jiang JS, Yu C. 2018. HOTAIR alleviates ox-LDL-induced inflammatory response in Raw264.7 cells via inhibiting NF-kB pathway. Eur Rev Med Pharmacol Sci. 22(20), 69916998.

24. Kattoor AJ, Pothineni NVK, Palagiri D, Mehta JL. 2017. Oxidative Stress in Atherosclerosis. Curr Atheroscler Rep. 19(11), 42.

25. Li T, Wu YN, Wang H, Ma JY, Zhai SS, Duan J. 2020. Dapk1 improves inflammation, oxidative stress and autophagy in LPS-induced acute lung injury via p38MAPK/NF-KB signaling pathway. Mol Immunol. 120, 13-22.

26. Nie N, Bai C, Song S, Zhang Y, Wang B, Li Z. 2020. Bifidobacterium plays a protective role in TNF-ainduced inflammatory response in Caco-2 cell through NF-KB and p38MAPK pathways. Mol Cell Biochem. 464(1-2), 83-91.

27. Lawrence T. 2009. The nuclear factor NF-kappaB pathway in inflammation. Cold Spring Harb Perspect Biol. 1(6), a001651.

28. Iqubal A, Iqubal MK, Sharma S, Ansari MA, Najmi AK, Ali SM, Ali J, Haque SE. 2019. Molecular mechanism involved in cyclophosphamide-induced cardiotoxicity: Old drug with a new vision. Life Sci. 218, 112-131.

29. Martin M, Fornecker LM, Marcellin L, Mousseaux E, Hij A, Snowden JA, Farge D, Martin T. 2017. Acute and fatal cardiotoxicity following high-dose cyclophosphamide in a patient undergoing autologous stem cell transplantation for systemic sclerosis despite satisfactory cardiopulmonary screening. Bone Marrow Transplan 52(12), 1674-1677.

30. Wang YM, Du GQ. 2016. Glycyrrhizic acid prevents enteritis through reduction of NF-kB p65 and p38MAPK expression in rat. Mol Med Rep. 13(4), 3639-46.

31. Cuker A, Arepally GM, Chong BH, et al. 2018. American Society of Hematology 2018 guidelines for management of venous thromboembolism: heparin-induced thrombocytopenia. Blood Adv. 2(22), 3360-3392.

32. Zhang Z, Li Z, Li J, Liu L. 2018. Effects of Natural Hirudin and Low Molecular Weight Heparin in Preventing Deep Venous Thrombosis in Aged Patients with Intertrochanteric Fracture. Sci Rep. 8(1), 8847.

33. Deng F, Zhang J, Li Y, et al. 2019. Hirudin ameliorates immunoglobulin A nephropathy by inhibition of fibrosis and inflammatory response. Ren Fail. 41(1), 104-112.

34. Zhang H, Xu F, Cui LQ, Yang D, Zheng YH. 2020. Methods for Creating Medium and Large Animal Models of Peripheral Atherosclerosis]. Zhongguo Yi Xue Ke Xue Yuan Xue Bao 42(2), 257-262.

35. Shen W, Shi HM, Fan WH, Luo XP, Jin B, Li Y. 2011. The effects of simvastatin on angiogenesis: studied by an original model of atherosclerosis and acute myocardial infarction in rabbit. Mol Biol 
Rep. 38(6), 3821-8.

36. Wang C, Liu J, Guo F, Ji Y, Liu N. 2009. Endothelin-1 induces the expression of C-reactive protein in rat vascular smooth muscle cells. Biochem Biophys Res Commun. 389(3), 537-42.

37. Jellinger PS, Handelsman Y, Rosenblit PD, Bloomgarden ZT, Fonseca VA, Garber AJ, Grunberger G, Guerin CK, Bell DSH, Mechanick JI, Pessah-Pollack R, Wyne K, Smith D, Brinton EA, Fazio S, Davidson M. 2017. AMERICAN ASSOCIATION OF CLINICAL ENDOCRINOLOGISTS AND AMERICAN COLLEGE OF ENDOCRINOLOGY GUIDELINES FOR MANAGEMENT OF DYSLIPIDEMIA AND PREVENTION OF CARDIOVASCULAR DISEASE. Endocr Pract. 23(Suppl 2), 1-87 (2017).

38. Han X, Chen H, Zhou J, Steed H, Postovit LM, Fu Y. 2018. Pharmacological Inhibition of p38 MAPK by SB203580 Increases Resistance to Carboplatin in A2780cp Cells and Promotes Growth in Primary Ovarian Cancer Cells. Int J Mol Sci. 19(8), 2184.

39. Gao X, Li N, Zhang J. 2019. SB203580, a p38MAPK inhibitor, attenuates olfactory dysfunction by inhibiting OSN apoptosis in AR mice (activation and involvement of the p38 mitogen-activated protein kinase in olfactory sensory neuronal apoptosis of OVA-induced allergic rhinitis). Brain Behav. 9(6), e01295.

40. Zelová H, Hošek J. 2013. TNF-a signalling and inflammation: interactions between old acquaintances. Inflamm Res. 62(7), 641-651.

41. Scheller J, Chalaris A, Schmidt-Arras D, Rose-John S. 2011. The pro- and anti-inflammatory properties of the cytokine interleukin-6. Biochim Biophys Acta. 1813(5), 878-888.

42. Lopez-Castejon G, Brough D. 2011. Understanding the mechanism of IL-1 $\beta$ secretion. Cytokine Growth Factor Rev. 22(4), 189-95 (2011).

43. Bourque SL, Davidge ST, Adams MA. 2011. The interaction between endothelin-1 and nitric oxide in the vasculature: new perspectives. Am J Physiol Regul Integr Comp Physiol. 300(6), R1288-95.

44. Liu J, Liu L, Sun J, Luo Q, Yan C, Zhang H, Liu F, Wei Y, Dong J. 2020. Icariin Protects Hippocampal Neurons From Endoplasmic Reticulum Stress and NF-KB Mediated Apoptosis in Fetal Rat Hippocampal Neurons and Asthma Rats. Front Pharmacol 10, 1660.

45. Lv H, Zhang S, Hao X. 2020. Swainsonine protects H9c2 cells against lipopolysaccharide-induced apoptosis and inflammatory injury via down-regulating miR-429. Cell Cycle 19(2), 207-217 (2020).

46. Nežić L, Amidžić L, Škrbić R, Gajanin R, Nepovimova E, Vališ M, Kuča K, Jaćević V. 2019. Simvastatin Inhibits Endotoxin-Induced Apoptosis in Liver and Spleen Through Up-Regulation of Survivin/NFKB/p65 Expression. Front Pharmacol 10, 54 (2019).

47. Kim EK, Cho JH, Jeong AR, Kim EJ, Park DK, Kwon KA, Chung JW, Kim KO, Kim JH, Kim JH, Kim YJ. 2017. Anti-inflammatory effects of simvastatin in nonsteroidal anti-inflammatory drugs-induced small bowel injury. J Physiol Pharmacol. 68(1), 69-77.

\section{Figures}




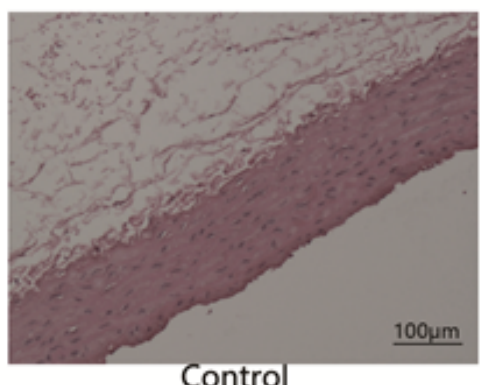

Control

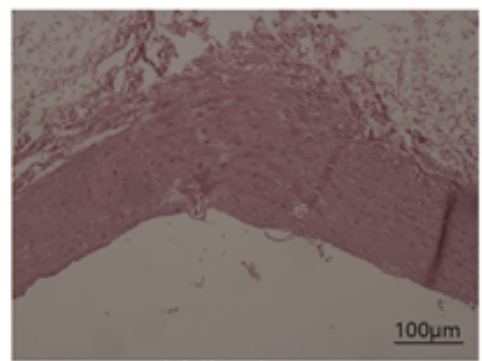

Middle-dose $\mathrm{r}-\mathrm{H}$

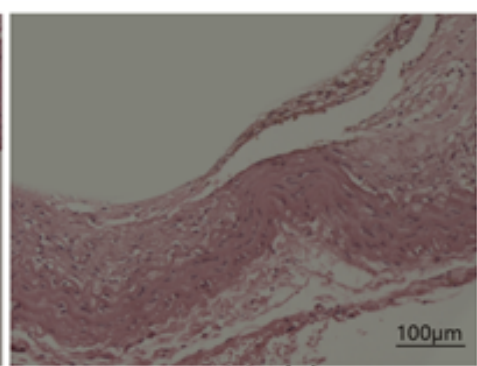

Model

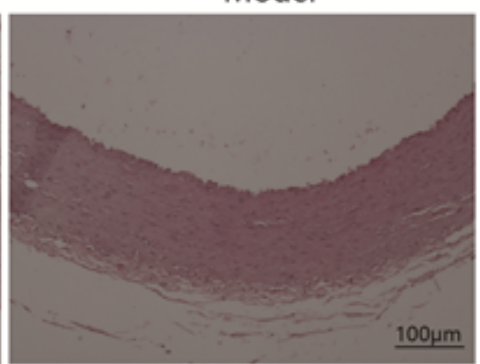

High-dose $\mathrm{r}-\mathrm{H}$

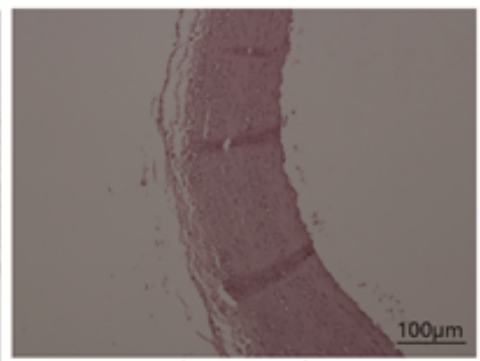

Simvastatin

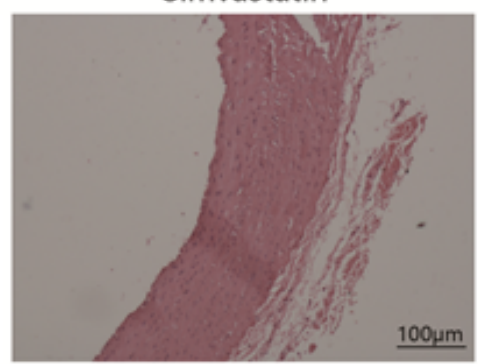

Inhibitor

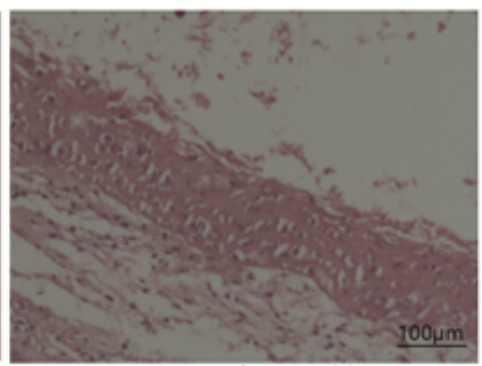

Low-dose $\mathrm{r}-\mathrm{H}$

\section{Figure 1}

HE staining of the rat common carotid artery (magnification, $\times 200$ )

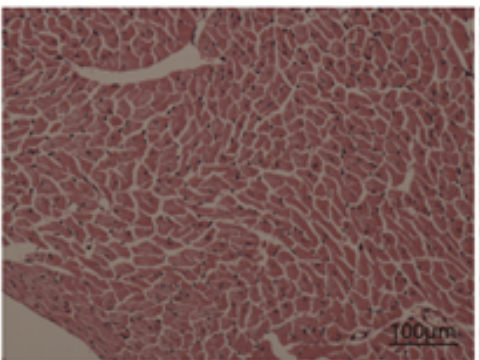

Control

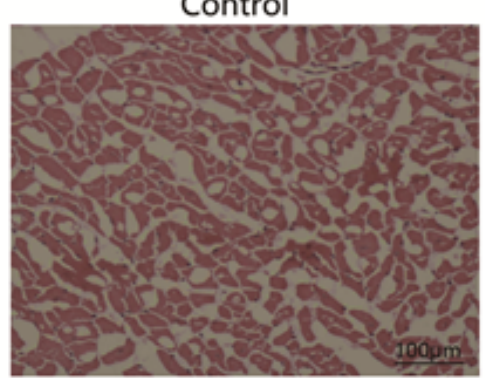

Middle-dose $\mathrm{r}-\mathrm{H}$

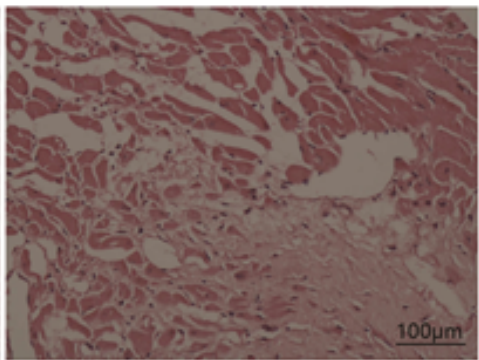

Model

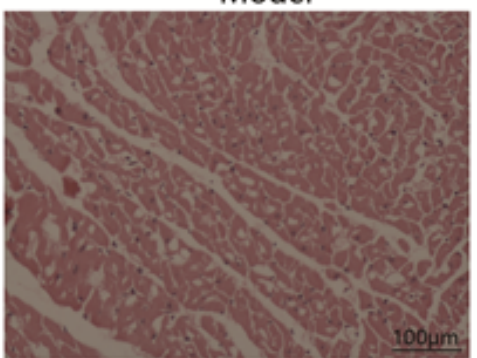

High-dose $\mathrm{r}-\mathrm{H}$

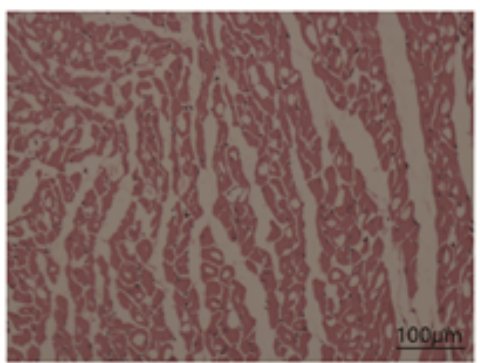

Simvastatin

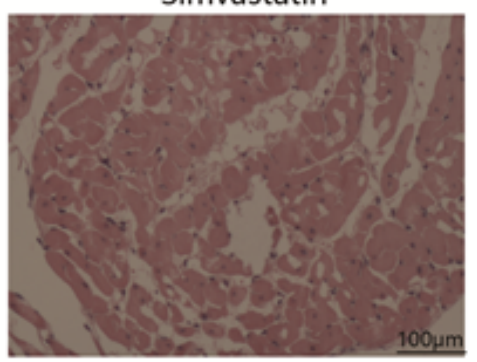

Inhibitor

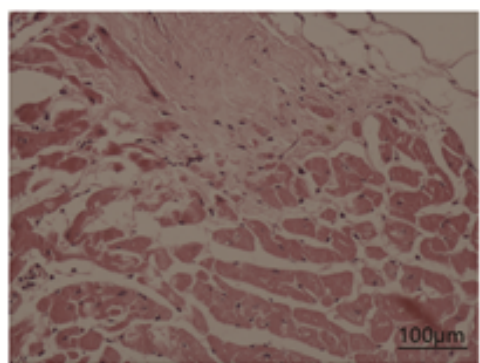

Low-dose r-H

\section{Figure 2}

HE staining of rat myocardial tissue (magnification, $\times 200$ ) 


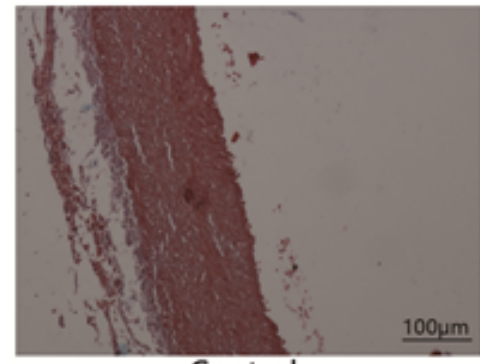

Control

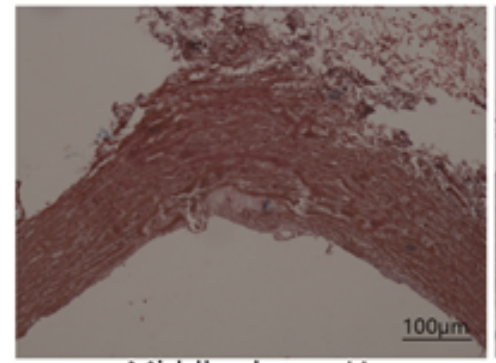

Middle-dose $\mathrm{r}-\mathrm{H}$

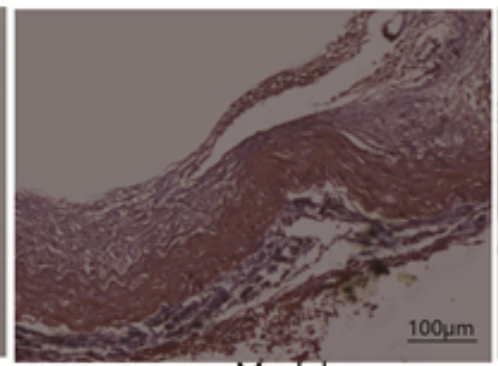

Model

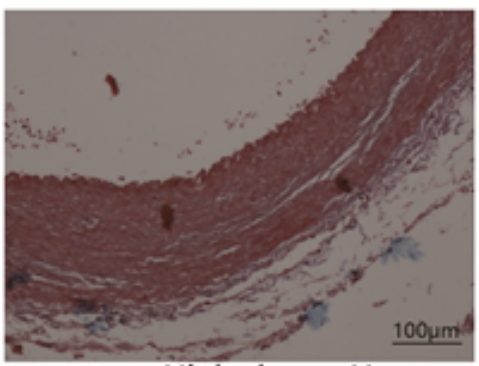

High-dose $\mathrm{r}-\mathrm{H}$

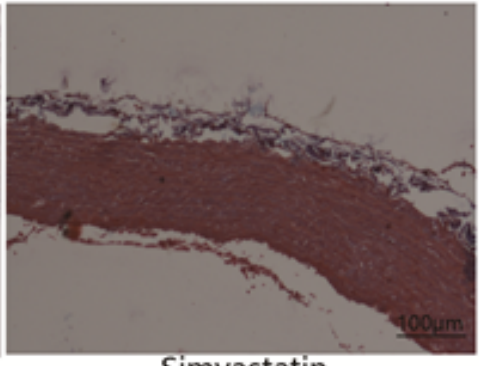

Simvastatin

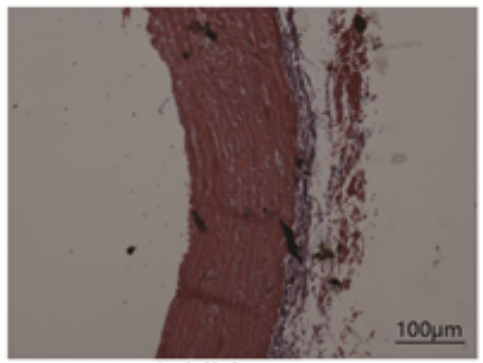

Inhibitor

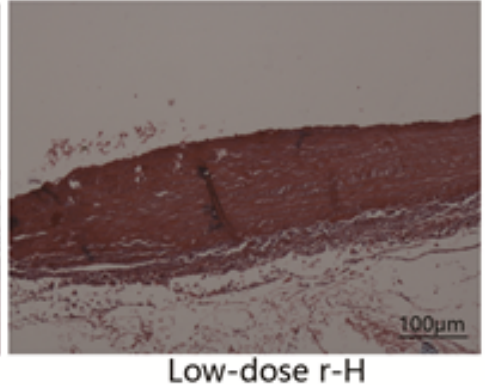

Low-dose $\mathrm{r}-\mathrm{H}$

Figure 3

Masson staining of the rat common carotid artery (magnification, $\times 200$ )

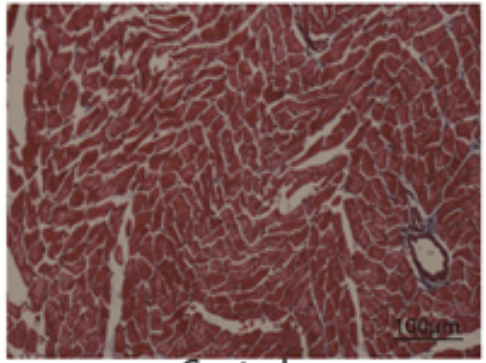

Contro

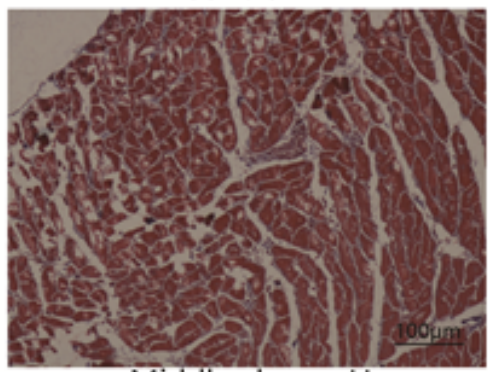

Middle-dose $\mathrm{r}-\mathrm{H}$

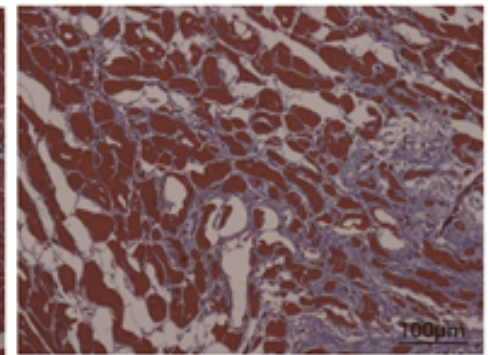

Model

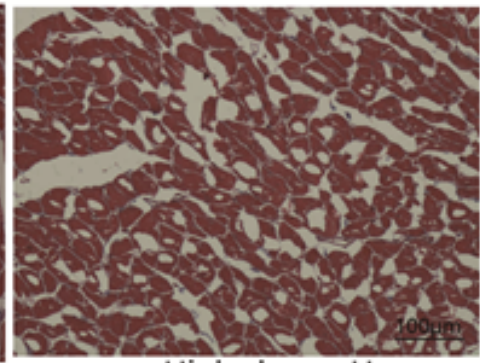

High-dose $\mathrm{r}-\mathrm{H}$

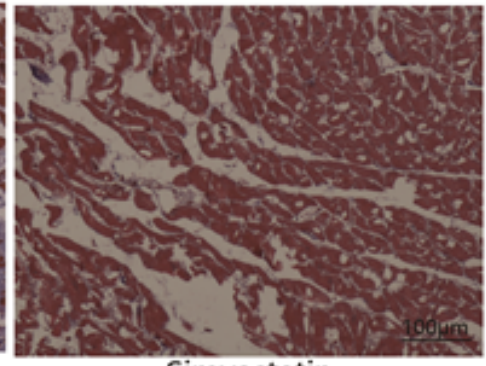

Simvastatin

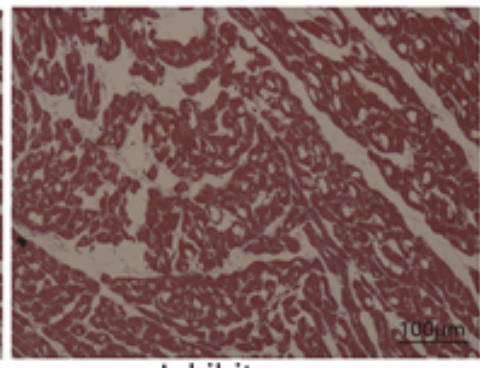

Inhibitor

\section{Figure 4}

Masson staining of rat myocardial tissue (magnification, $\times 200$ ) 


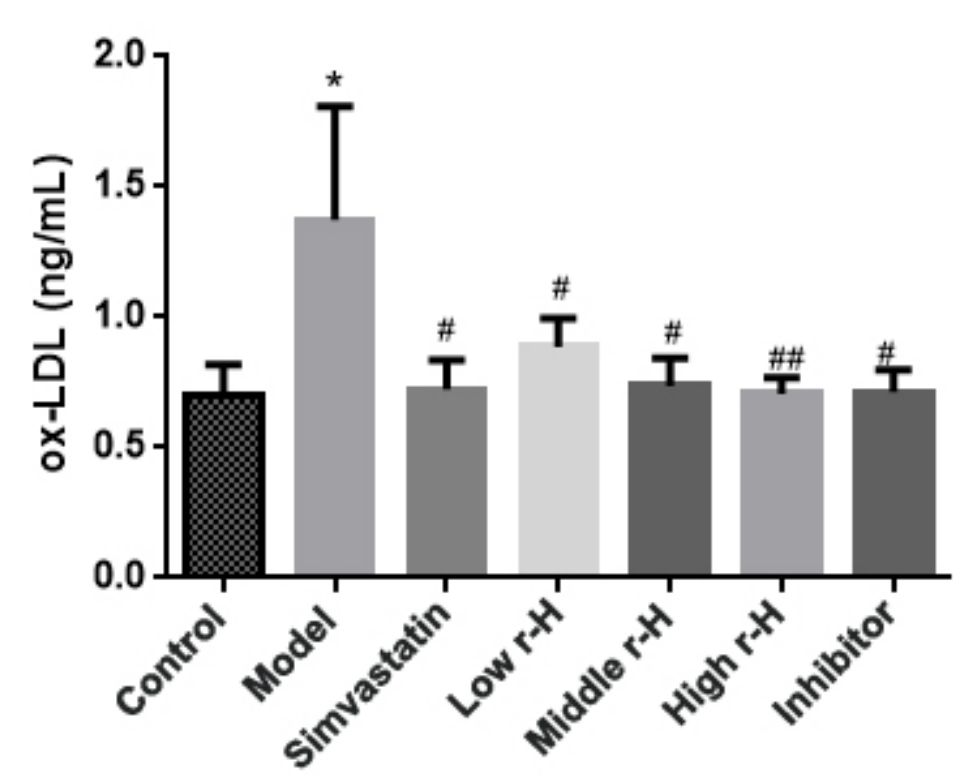

Figure 5

The level of ox-LDL in rats in the different groups, as determined by ELISA Compared with the control group, ${ }^{*}<<0.05$; compared with the model group, $\# \mathrm{P}<0.05$, \#\#P<0.01.

a

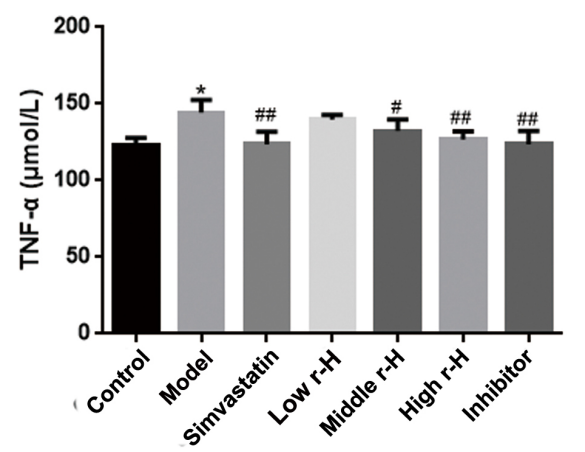

b

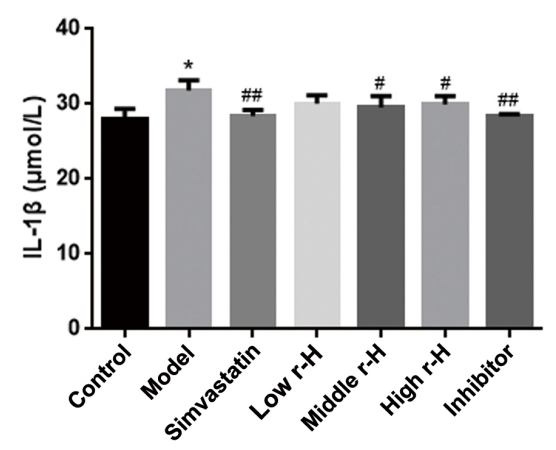

C

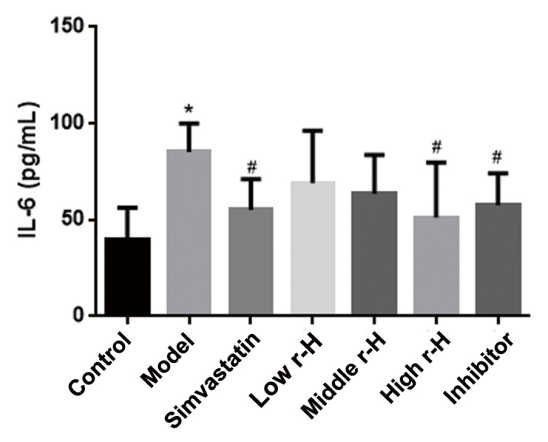

Figure 6

Effect of r-hirudin on the levels of TNF-a, IL-1 $1 \beta$ and IL- 6 in the sera of AS rats a. The level of TNF-a in rats from the different groups, as determined by ELISA. $b$. The level of IL-1 $\beta$ in rats in the different groups, as determined by ELISA. c. The level of IL- 6 in rats in the different groups, as determined by ELISA. Compared with the control group, ${ }^{*} \mathrm{P}<0.01$; compared with the model group, $\mathrm{QP} \otimes 0.05, \mathbb{\text { Q }} \mathrm{P}<0.01$. 
a

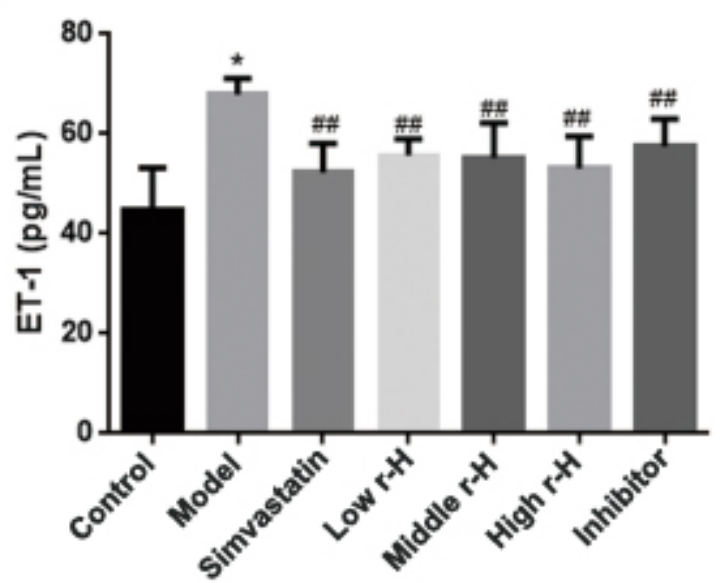

b

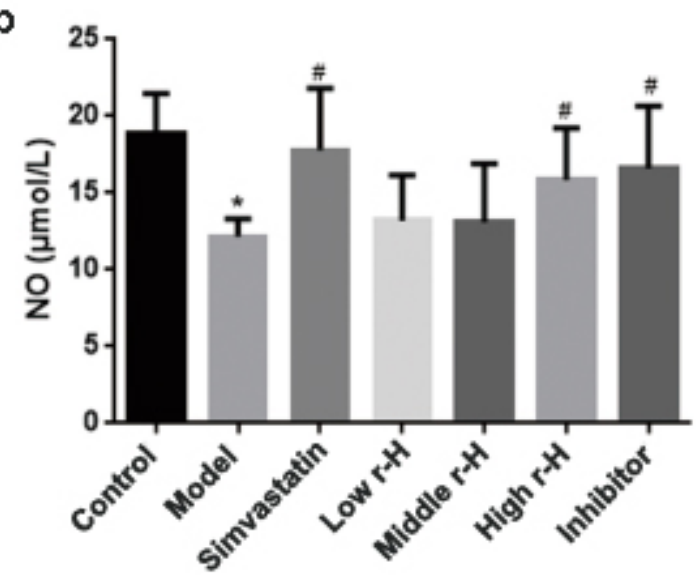

Figure 7

Effect of r-hirudin on the levels of ET-1 and NO in the sera of AS rats a.The level of ET-1 in rats in the different groups, as determined by ELISA. b. The level of NO in rats in the different groups, as determined by ELISA. Compared with the control group, ${ }^{*}<<0.01$; compared with the model group, $\triangle P \otimes 0.05, \mathbb{\nabla} \mathrm{P}<0.01$. 
a

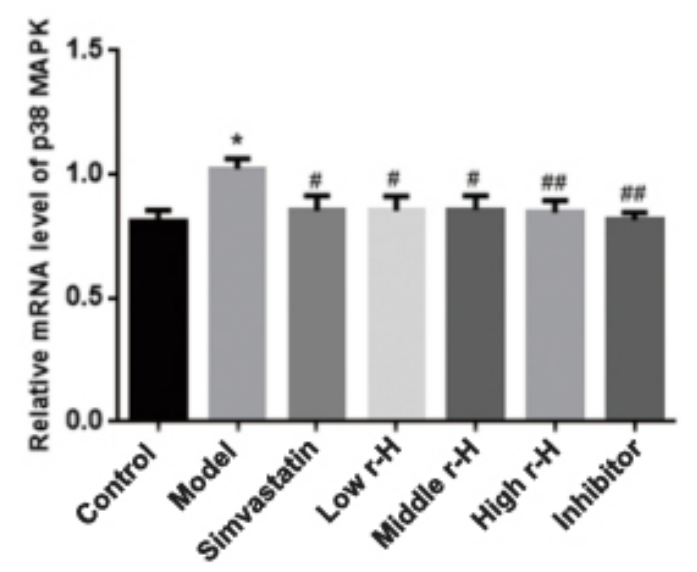

b

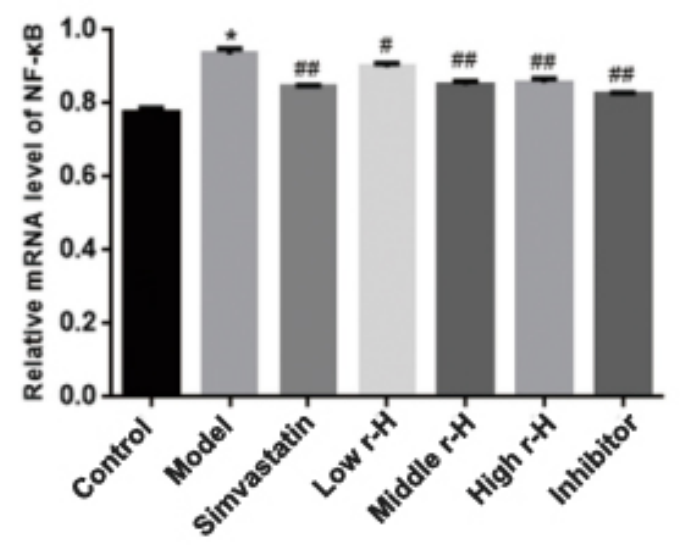

C
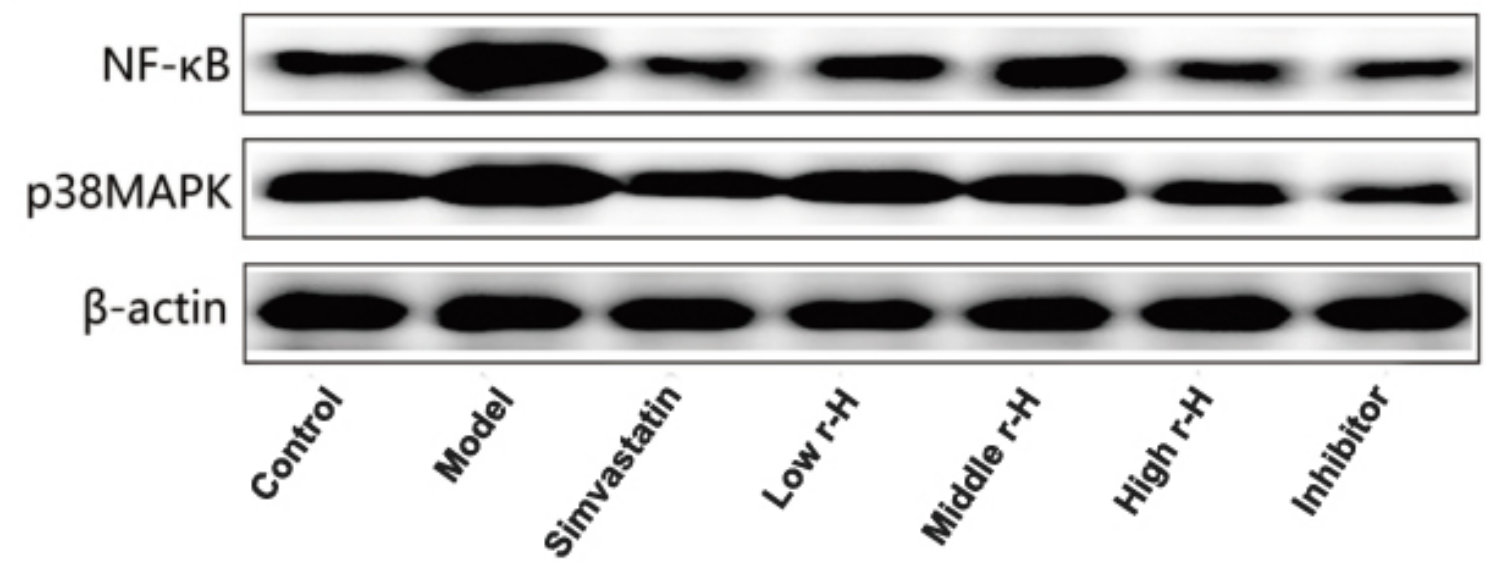

d

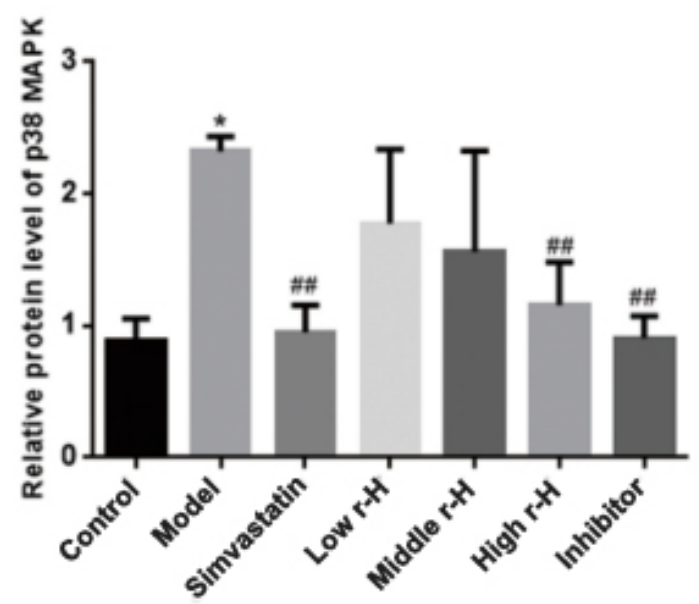

e

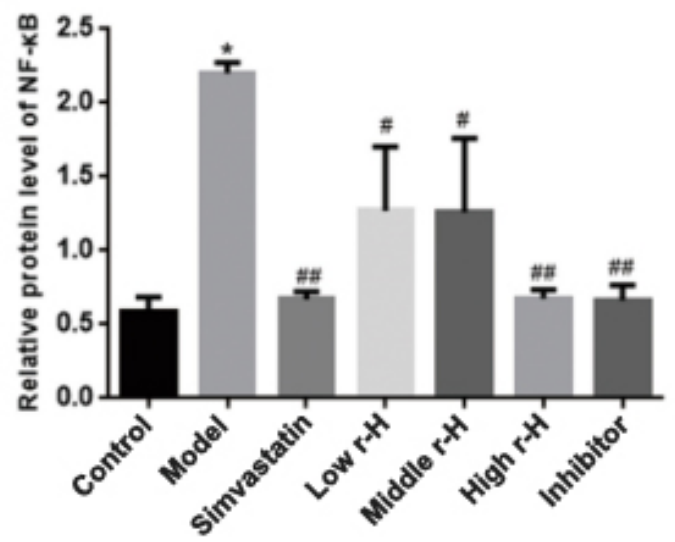

\section{Figure 8}

Effect of r-hirudin on the mRNA and protein expression levels of p38 MAPK and NF-KB in the common carotid arteries of AS rats $(a, b)$ The relative mRNA levels of p38 MAPK and NF-KB in rats in the different groups, as determined by qRT-PCR. (c-e) The protein expression of p38 MAPK and NF-kB in rats in the different groups, as determined by Western blotting. Compared with the control group, ${ }^{*} \mathrm{P}<0.01$; compared with the model group, $\# \mathrm{P}<0.05, \# \# P<0.01$. 
a

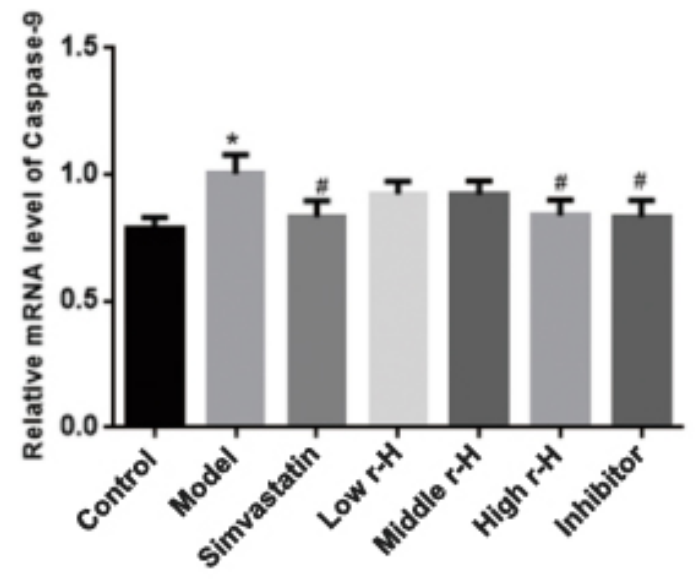

b

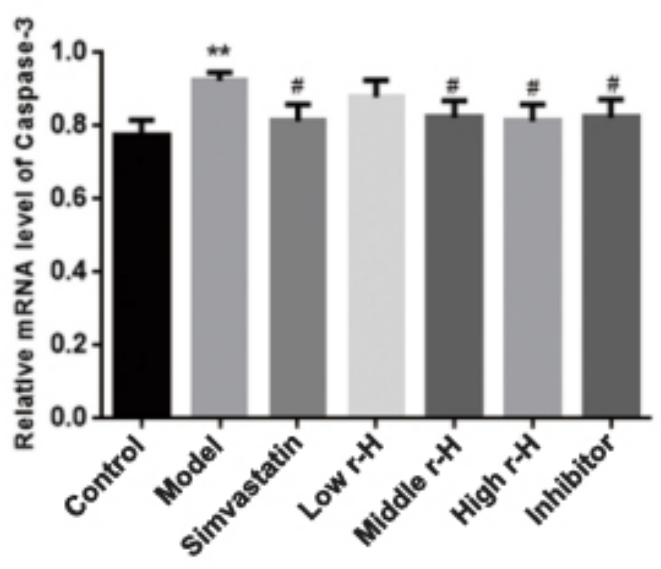

C
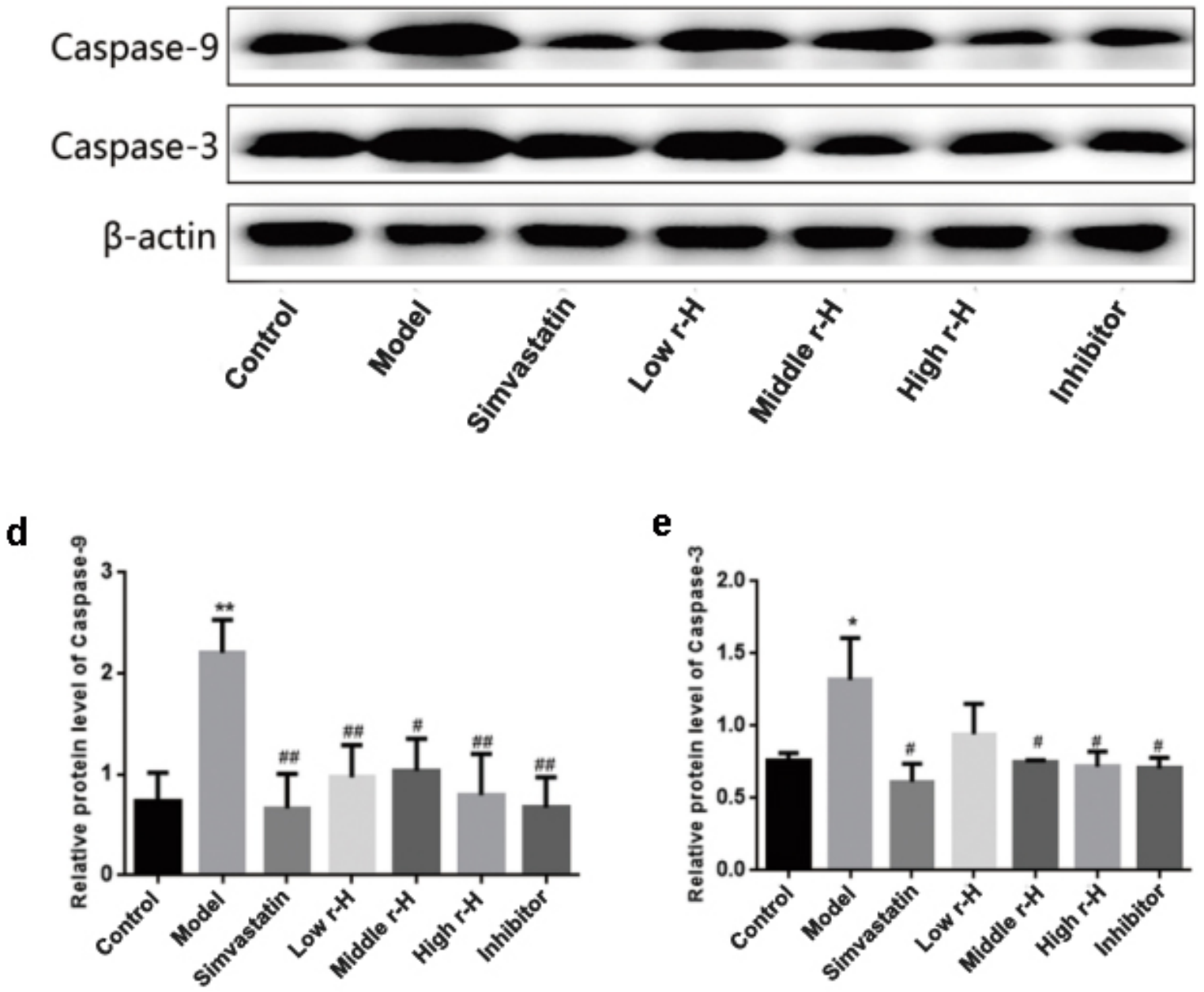

Figure 9

Effect of $r$-hirudin on the mRNA and protein expression levels of Caspase-9/Caspase- 3 in the common carotid arteries of AS rats $(a, b)$ The relative mRNA level of Caspase-9/Caspase-3 in rats in the different groups, as determined by qRT-PCR. (c-e) The protein expression of Caspase-9/Caspase- 3 in rats in the different groups, as determined by Western blotting. Compared with the control group, ${ }^{*} P<0.05,{ }^{*} P<0.01$; compared with the model group, $\# \mathrm{P}<0.05$, \#\#P<0.01. 